Florida International University FIU Digital Commons

\title{
Collective Equipoise, Disappointment and the Therapeutic Misconception: On the Consequences of Selection for Clinical Research
}

Peter Thompson

Department of Economics, Florida International University, thompsop@fiu.edu

Margaret M. Byrne

Department of Epidemiology and Public Health, University of Miami

Follow this and additional works at: https://digitalcommons.fiu.edu/economics_wps

\section{Recommended Citation}

Thompson, Peter and Byrne, Margaret M., "Collective Equipoise, Disappointment and the Therapeutic Misconception: On the Consequences of Selection for Clinical Research" (2005). Economics Research Working Paper Series. 75.

https://digitalcommons.fiu.edu/economics_wps/75 


\title{
Collective Equipoise, Disappointment and the Therapeutic Misconception: On the Consequences of Selection for Clinical Research
}

\author{
Margaret M. Byrne* \\ Department of Epidemiology and Public Health \\ University of Miami \\ Peter Thompson** \\ Department of Economics \\ Florida International University
}

June 2005

\begin{abstract}
Private information induces individuals to self-select as subjects into clinical research trials, and it induces researchers to select which trials they conduct. We show that selection can induce ex ante therapeutic misconception and ex post disappointment among research subjects; and it undermines it the rationale of collective equipoise as an ethical basis for clinical trials. Selection provides a reason to make non-trivial payments to subjects and it implies that researchers should not design experiments to maximize statistical power.
\end{abstract}

* Corresponding author: Highland Professional Building, Room 200J, 1801 N.W. 9th Avenue, Miami, FL 33136; tel: 305-243-3482; fax 305-243-5544 (email: mbyrne2@med.miami.edu). ** Department of Economics, Florida International University, Miami, FL 33199; tel: 305-348-6031 (email: peter.thompson2@fiu.edu).

JEL Classification: I1 


\section{Introduction}

In empirical work, selection bias is consistently one of the greatest challenges to researchers. It undermines the inferences we make in several ways: it makes treatments endogenous, it affects who we observe in a sample and who we do not, and it even affects the research we choose to undertake. Clinical trials have long dealt with the first consequence of selection bias by using trials in which treatments are allocated at random. But there has been little progress in dealing with other types of selection. In this paper we explore the consequences of two types of selection in clinical trials: of subjects into trials on the basis of private information, and of researchers into trials on the basis of information unobserved by the profession at large.

These selection problems have important consequences for some deeply-held attitudes about which trials are ethical to conduct, and the manner in which they should be conducted. In particular, selection by subjects generates ex ante therapeutic misconception and ex post disappointment about the personal benefit from participating in trials, and it provides a rationale for making payments to subjects that are likely to be much larger than has been the norm. Selection of researchers undermines the rationale for collective equipoise coexisting with trials that are designed to maximize statistical power. If collective equipoise is to be maintained as an ethical basis for deciding which trials can be conducted, then it is unethical to maximize statistical power; if maximizing statistical power is to be maintained as the basic criterion for experimental design, then collective equipoise is unethical.

Although selection by subjects and by researchers are clearly closely related issues, it is useful to explore their implications separately. In the introduction, we begin the paper as we mean to go on. In Section A we discuss further the 
problem of selection by subjects, the therapeutic misconception, and disappointment. In Section $\mathrm{B}$ we discuss selection by researchers and the collective equipoise problem.

A. Self-Selection by Subjects, the Therapeutic Misconception, and Disappointment

It is widely understood among members of the profession that the ethics and practice of clinical research and ordinary clinical care are fundamentally different. When subjects do not understand this, it is known as the therapeutic misconception (Appelbaum, Roth and Lidz [1982]). Therapeutic misconceptions arise when subjects transfer their expectation that physicians will act in the patient's best interest from a clinical setting to a research setting (Appelbaum [2002]); because they do not understand the concept of randomization (Kerr et al. [2004]); because they do not understand the constraints imposed on treatments offered during trials (Dresser [2002]); or because they have an overwhelming psychological need to believe that participation will yield personal benefits (Appelbaum et al. [1982]). The combined effect of these disparate influences is that subjects' subjective expectations of the personal benefit of participation exceeds the mathematical expectations.

The therapeutic misconception conflicts directly with the principle of respect for persons as expressed in the requirement of informed consent from voluntary participants. Nonetheless, as Dresser [2002] has noted, researchers have in practice tolerated therapeutic misconceptions as an inevitable consequence of conducting trials. Some have gone so far as to promote them (e.g. Truog et al. [1999]). However, a number of proposals have been offered to attenuate the therapeutic misconception, including the use of a "neutral discloser" (Appelbaum et al. [1982]), changes to consent forms (Appelbaum [1996], Moreno et al. [1998]), changes in advertising (Miller and Shorr [1999]), changes in the way physicians 
recruit subjects (Kass et al. [1996]), and requiring physicians to disclose to their patients payments they are receiving for recruiting subjects (Berg [1997]).

In many practical settings, these proposed solutions can only make limited progress. When the efficacy of an experimental treatment varies among individuals, self-selection into trials by individuals who are at least partiallyinformed ensures that subjects correctly expect to reap a personal benefit. However, under two reasonable scenarios, subjects that do not suffer therapeutic misconception in the original sense proposed by Appelbaum et al. [1982] will continue to expect, or give the appearance of expecting, personal benefits that exceed the true expectation.

For both scenarios we consider a setting in which researchers and potential subjects have prior beliefs about the individual benefit that will arise from participating in an experiment. The priors for both groups have zero mean. When a potential subject is given an opportunity to join a trial, she also receives a private signal about the personal benefit of participation. With the signal she updates her beliefs, and joins the trial if her posterior mean is positive. In the first scenario, the potential subject's beliefs are formed efficiently (i.e. by Bayesian means), so that, whether or not she decides to join, her subjective mean is equal to the mathematical expectation conditional on the signal. However, researchers do not observe the private signal because they have only limited information about the subject, and so their belief remains unchanged from the prior. ${ }^{1}$ When researchers subsequently compare their expectation about the

\footnotetext{
${ }^{1}$ Subjects may have found that existing treatments have not worked for them, or that they had unusually severe side effects. Chard and Lilford [1998] point out that most treatments involve a vector of characteristics and how these are weighted depends on
} 
personal benefit with the expectations of their subjects, they conclude that every single subject suffers from the therapeutic misconception. In reality, no one does.

In the second scenario, potential subjects are not efficiently Bayesian. The key assumption we make is that individuals are overconfident about the accuracy of signals they receive, in the sense that they believe noisy signals indicating the benefit from participation to be more accurate than they really are. ${ }^{2}$ In this scenario, subjects revise their beliefs too much in response to the signal. Although the average subjective posterior mean of all individuals is unbiased, self-selection into trials by individuals that received a positive signal implies that every subjects' posterior means exceeds the true conditional expectation. Researchers, whether they observe the signal or not, will again conclude that subjects suffer from the therapeutic misconception. In this case they are correct.

The second scenario is clearly of more concern. When individuals are overconfident they are not capable of providing informed consent, even though they make use of all information available to them. Absent conscious intervention to tackle the problem, the therapeutic bias vanishes only at the extremes when subjects are either completely uninformed or perfectly informed. The former

patient preferences.

${ }^{2}$ There is an extensive and convincing psychological literature supporting the assumption of overconfidence about the accuracy of private information. It has been found in numerous experimental settings (Lichtenstein, Fischhoff, and Phillips [1982], Yates [1990]), and in many professional fields (see Odean [1998] and Barber and Odean [2001] for extensive references). The idea has since been fruitfully applied to applications in finance (De Long et al. [1991], Daniel, Hirshleifer, and Subrahmanyam [1998], Kyle and Wang [1997], Odean [1998], entrepreneurship (Klepper and Thompson [2005]), and auction theory (Compte [2004]). 
extreme hardly seems a desirable way to eliminate the therapeutic bias; the latter hardly seems feasible.

It is important to distinguish between the therapeutic misconception and the better-known concept of the winner's curse. We have interpreted the therapeutic misconception as being a state in which a subject's expectation exceeds the mathematical expectation. The winner's curse, which we will call "disappointment" here to distinguish it from settings in auction theory, exists when a subject's expectation exceeds the realized benefit of participation. We will show that selection alone induces disappointment on average. However, overconfidence exacerbates the problem. ${ }^{3}$

Section II of the paper formalizes these ideas. We then show that in many settings a simple solution is to offer a significant monetary payment for participation. This finding is incompatible with the widespread practice of offering only token compensation, apparently because of concerns that sizeable payments might be "coercive", or might undermine altruistic motivations to participate. However, we are not the first to propose large payments to tackle the therapeutic misconception. Dickert and Grady [1999] have argued that payments would serve to "remind" subjects that participation provides service to others rather than to the subject. While our case for payments is different, it complements their proposal.

\section{B. Self-Selection by Researchers and Clinical Equipoise}

The prevailing view among ethicists is that clinical trials should be

${ }^{3}$ Compte [2004] has employed a similar idea to generate the winner's curse from private value Vickrey auctions. 
conducted only under conditions of equipoise. Individual equipoise exists when a rational informed person has no preference between two treatments, say an established treatment and an experimental treatment. Collective equipoise exists when the profession as a whole has no clear preference for one treatment over another. Under collective equipoise, individuals may have distinct preferences but differing preferences approximately cancel each other out (Johnson, Lilford, and Brazier [1991], Gifford [1995]). The notion of equipoise underlies the 1964 Declaration of Helsinki, which prohibits the use of placebos when an effective treatment already exists, and it is a central concern of ethics committees (Kennedy [1991]).

A case has been made that trials should be conducted only in situations of "collective equipoise" (Freedman [1987]). It has also been argued that the Golden rule demands that individual equipoise holds for each physician suggesting research participation to his or her own patients (Chard and Lilford [1998]). Thus, both collective and individual equipoise have been indicated as essential for the ethical conduct of clinical trials.

Selection by researchers into trials undermines obtaining both collective and individual equipoise. Collective equipoise is based on public information, most notably the information revealed from the publication of the results of randomized trials. But individuals conducting these trials invariably have private information based on their specialized efforts in preliminary experimentation, and their ongoing research into the physiology or biochemistry of possible treatments. ${ }^{4}$ If ethical researchers select themselves into conducting promising

4 The claim that researchers have prior information is well-understood. For example Lilford [2003, p. 980] writes that "[s]ome evidence always exists before a randomized 
experiments, and they are better informed than the collective consciousness, then it must be the case that collective equipoise is consistent with a positive expected benefit of a new treatment.

Section III analyses the causes and consequences of selection by researchers. We assume that a researcher is faced with a sequence of research opportunities, but resource constraints prevent her from pursuing all of them. Collective equipoise requires that the benefit of innovative treatments (relative to a placebo or existing treatment, as appropriate) embodied in each of these opportunities has prior mean zero. ${ }^{5}$ Accompanying each opportunity, the researcher receives a private signal about the expected benefit. In this setting, the researcher only initiates projects for which her posterior mean is strictly positive. That is, if there is collective equipoise, there cannot be individual equipoise. To restore individual equipoise, ethics committees must be willing to approve trials on innovative treatments for which the (collective) prior mean is negative.

We also explore one important implication of selection. If collective equipoise is maintained as an ethical criterion, selection by better-informed researchers into experiments on treatments for which there is collective equipoise undermines the

clinical trial is done: in vitro and animal experiments, the same treatment in other diseases, similar treatments in the same disease, and perhaps even randomized clinical trials done elsewhere. Thus clinicians have some idea of what treatments might accomplish, even in advance of a trial." The consequences of this prior information for selection does not appear to be so well understood.

${ }^{5}$ This is an excessively precise interpretation of collective equipoise convenient for modeling. It ignores what Chard and Lilford [1998, p.898)] have called the "fuzzy boundary of appreciation of numeric concepts inherent in human behavior." 
case for experimental designs that maximize statistical power. Instead, experimental designs should reflect prior information held by researchers, trading statistical power against the expected net benefits for subject participants. How we should go about doing this depends critically on how we choose to weight the short-term benefits for participants against possible future benefits for society. We do not attempt to resolve that issue in this paper, which is outside the scope of formal economic analysis. However we do explore the factors that affect optimal experimental design conditional on any given set of weights.

\section{Self-Selection by Subjects}

When an individual is faced with an opportunity to participate in a trial, it comes accompanied by a signal, $\zeta$, which is positively correlated with the private benefit of participation. If she chooses to participate in the trial she receives a benefit $b$. The benefit has unconditional distribution $F(b)$, with zero expectation and domain $[\underline{b}, \bar{b}]$. Clearly, for a non-degenerate distribution we must have $\underline{b}<0$ and $\bar{b}>0$. The individual's subjective belief about $b$ after observing the signal is given by the conditional distribution, $F(b \mid \zeta)$, which is decreasing in $\zeta$ for all $b$. Hence, $E[b \mid \zeta]$ is increasing in $\zeta$. The subjective posterior distribution and mean may differ from the Bayesian posteriors, which we denote by $F^{*}(b \mid \zeta)$ and $E^{*}[b \mid \zeta]$.

The individual will choose to participate in the trial if she received a signal such that $E[b \mid \zeta] \geq 0$. If $\hat{\zeta}$ denotes the signal that satisfies $E[b \mid \hat{\zeta}]=0$, all subjects must have received signals, $\zeta \geq \hat{\zeta}$. Let $G(\zeta)$ denote the distribution of the signal, and let $G^{*}(\zeta \mid b)$ denote the (correct) conditional distribution. ${ }^{6}$ Then

6 Letting lower case letters denote the corresponding densities, Bayes' rule gives 
the average belief of all individuals with true benefit $b$, is

$$
\int_{-\infty}^{\infty} E[b \mid \zeta] d G^{*}(\zeta \mid b)
$$

while the average belief of all subjects with true benefit $b$ is

$$
\frac{1}{1-G^{*}(\hat{\zeta} \mid b)} \int_{\hat{\zeta}}^{\infty} E[b \mid \zeta] d G^{*}(\zeta \mid b) \text {. }
$$

Building on Compte [2004], we make the following assumptions:

$$
\text { A.1 } \quad E^{*}[\zeta \mid b]=b,
$$

A.2 $\quad E[b \mid \zeta]=\lambda(\zeta) E^{*}[b \mid \zeta]$,

$$
\text { A.3 } \lambda(\zeta) \geq 1 \forall \zeta \neq 0 ; \lambda(\zeta)=\lambda(-\zeta) \text {. }
$$

Assumption A.1 says that the signal is unbiased. Assumption A.2 states that the (possibly erroneous) subjective posterior mean can be expressed as a proportion of the correct mathematical expectations, where the factor of proportionality may depend on the observable $\zeta$ but not on the unobservable $b$. Assumption A.3 states that deviations of the signal from the prior in either direction induce the same degree of bias between the subjective and objective conditional means, which ensures we do not introduce therapeutic misconception by construction. If individuals are Bayesian, then $\lambda(\zeta)=1 \forall \zeta$. If individuals are overconfident, then $\lambda(\zeta)>1 \forall \zeta \neq 0$. Given these assumptions, $\hat{\zeta}=0$.

\section{A. The Therapeutic Misconception}

It is now easy to evaluate the extent of apparent or real therapeutic $g^{*}(\zeta \mid b)=f^{*}(b \mid \zeta) g(\zeta) / f(b)$. 
misconception under the two scenarios introduced in Section IA. Consider first the scenario in which individuals are Bayesian, but their signal is not observed by the researcher. ${ }^{7}$ Then the researcher believes $E[b]=0$, while the average posterior mean of subjects is

$$
\frac{1}{1-G^{*}(0 \mid b)} \int_{0}^{\infty} E^{*}[b \mid \zeta] d G^{*}(\zeta \mid b)>0 .
$$

Thus, the researcher concludes there is therapeutic misconception. However, (3) is the correct conditional expectation, so the appearance of therapeutic misconception is spurious. Concerns about the therapeutic misconception arise in this case only because outside observers fail to acknowledge that subjects selfselect.

In the second scenario, with overconfident individuals, average therapeutic misconception for any given $b$ is given by

$$
\begin{aligned}
\mu(b) & =\frac{1}{1-G^{*}(0 \mid b)} \int_{0}^{\infty} E[b \mid \zeta]-E^{*}[b \mid \zeta] d G^{*}(\zeta \mid b) \\
& =\frac{1}{1-G^{*}(0 \mid b)} \int_{0}^{\infty}(\lambda(\zeta)-1) E^{*}[b \mid \zeta] d G^{*}(\zeta \mid b) \\
& >0 .
\end{aligned}
$$

In this case, the apparent therapeutic misconception is real.

In general, the function $\lambda(\zeta)$ will depend on both the prior distribution of $b$ and the distribution of the signal. To investigate how these distributions affect the severity of the therapeutic misconception, it is useful to turn to a specific

${ }^{7}$ We can readily extend the analysis to allow researchers to observe the signals with noise. The same implications follow, however. 
example. Hence, assume that $F(b)$ is normal with mean zero and variance $\sigma_{b}^{2},{ }^{8}$ and that $\zeta=b+\varepsilon$, where $\varepsilon \sim N\left(0, \sigma_{\varepsilon}^{2}\right)$. Then, given a signal, $\zeta$, the conditional expectation is

$$
E^{*}[b \mid \zeta]=\frac{\sigma_{b}^{2} \zeta}{\sigma_{b}^{2}+\sigma_{\varepsilon}^{2}}
$$

We assume that the subjective posterior mean is given by

$$
E[b \mid \zeta]=\frac{\sigma_{b}^{2} \zeta}{\sigma_{b}^{2}+\lambda^{-1} \sigma_{\varepsilon}^{2}}
$$

for some constant $\lambda>1$. Conditional on $b, G^{*}(\zeta \mid b) \sim N\left(b, \sigma_{\varepsilon}^{2}\right)$, while the unconditional distribution of the signal is $G(\zeta) \sim N\left(0, \sigma_{b}^{2}+\sigma_{\varepsilon}^{2}\right)$. Then, the average therapeutic misconception for all individuals with true benefit $b$ and entry threshold $\hat{\zeta}$ is

$$
\mu(b)=\frac{(\lambda-1) \sigma_{b}^{2} \sigma_{\varepsilon}^{2}}{\left(1-G^{*}(\hat{\zeta} \mid b)\right)\left(\lambda \sigma_{b}^{2}+\sigma_{\varepsilon}^{2}\right)\left(\sigma_{b}^{2}+\sigma_{\varepsilon}^{2}\right)} \int_{\hat{\zeta}}^{\infty} \zeta d G^{*}(\zeta \mid b)>0,
$$

while the average therapeutic misconception over all subjects is

$$
\mu \equiv \int_{-\infty}^{\infty} \mu(b) d F(b)=\frac{(\lambda-1) \sigma_{b}^{2} \sigma_{\varepsilon}^{2}}{(1-G(\hat{\zeta}))\left(\lambda \sigma_{b}^{2}+\sigma_{\varepsilon}^{2}\right)\left(\sigma_{b}^{2}+\sigma_{\varepsilon}^{2}\right)} \int_{\hat{\zeta}}^{\infty} \zeta d G(\zeta)>0 .
$$

In this setting, the correct threshold is again $\hat{\zeta}=0$, but it is useful not to impose this for now so we can assess the effect of $\hat{\zeta}$ on the size of the therapeutic misconception. ${ }^{9}$

${ }^{8}$ That is, the interval $[\underline{b}, \bar{b}] \rightarrow(-\infty, \infty)$.

${ }^{9}$ For example, if there is a cost, $c$, (in time, travel, discomfort, etc.) of participating, then $\hat{\zeta}=\hat{\zeta}(c)$ is increasing in $c$. 

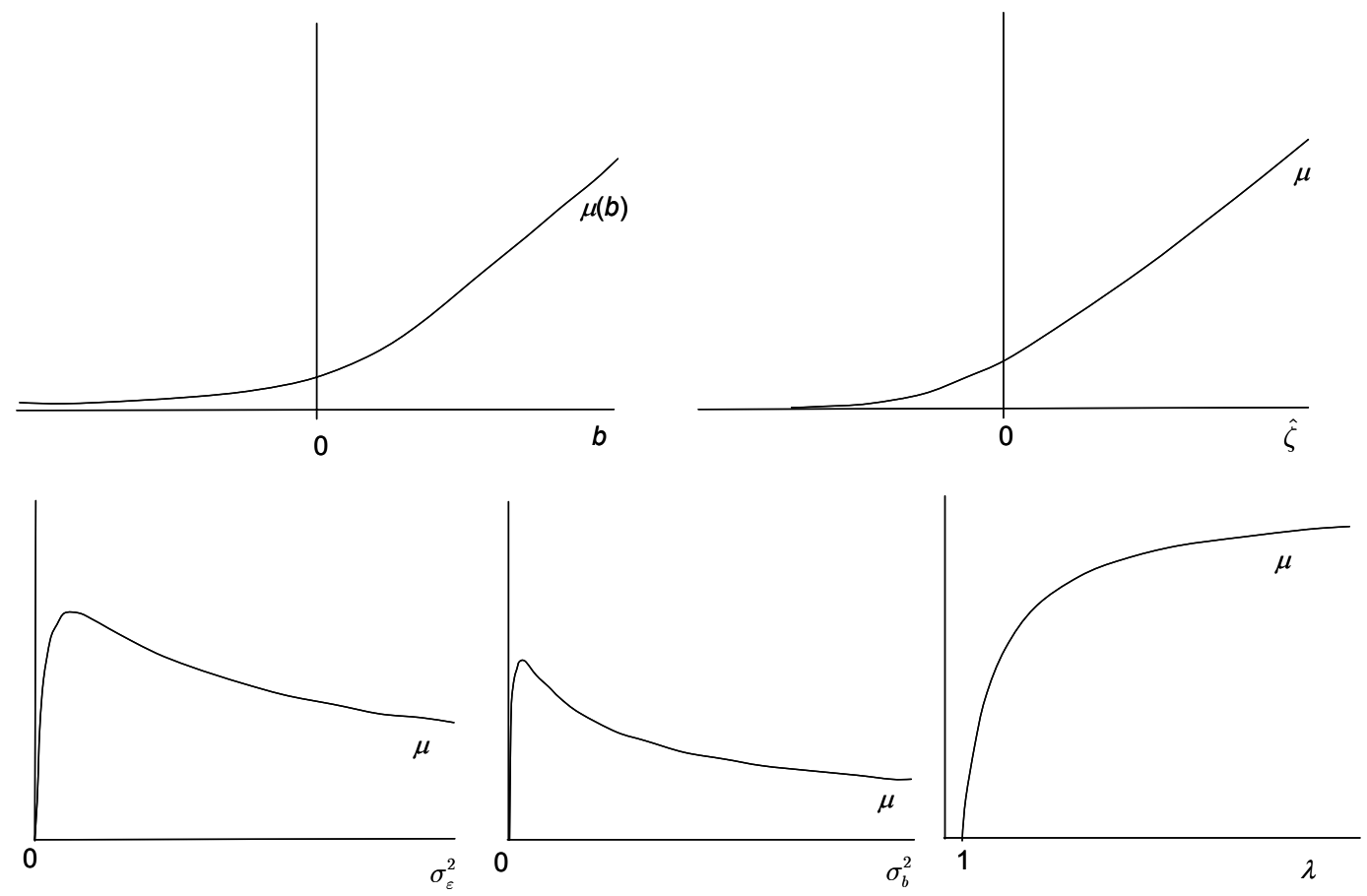

FiguRE 1. Comparative statics of average therapeutic misconception among subjects.

It is straightforward to assess the effects of parameter changes by evaluating (7) and (8). Figure 1 summarizes the effects with representative plots. The function $\mu(b)$ is monotonically increasing, and therapeutic misconception exists for all values of $b$. Somewhat surprisingly, then, the greater the true benefit for any given individual, the greater the therapeutic misconception. This arises because the term $E^{*}[b \mid \zeta]$ is stochastically increasing in $b$, so that $(\lambda(\zeta)-1) E^{*}[b \mid \zeta]$ is also stochastically increasing. Across all subjects, $\mu$ rises monotonically with $\hat{\zeta}$, reflecting the role of selection in creating the therapeutic misconception. Given that a personal cost, $c$, of participation raises $\hat{\zeta}$, the 
therapeutic misconception is greater in trials that impose a greater personal burden on the subject. The function $\mu$ also rises monotonically with $\lambda$, but it is has an upper bound given by $\lim _{\lambda \rightarrow \infty} \mu=2^{1 / 2} \sigma_{\varepsilon}^{2}\left(\sigma_{b}^{2}+\sigma_{\varepsilon}^{2}\right)^{-1 / 2} \pi^{-1 / 2}$. Hence, the effect of overconfidence is bounded. As confidence rises, so does the therapeutic misconception, but only until it attains the limit when subjects disregard the prior.

Finally, $\mu$ initially rises and then falls with increases in $\sigma_{b}^{2}$ and $\sigma_{\varepsilon}^{2}$; the numerical plots indicate that average therapeutic misconception is at its worst for modest values of $\sigma_{b}^{2}$ and $\sigma_{\varepsilon}^{2}$, in both cases declining asymptotically to zero at a very slow rate. When signals are accurate, (i.e. $\sigma_{\varepsilon}^{2} \rightarrow 0$ ), it is not possible to overweight the signal relative to the prior, for the simple reason that the correct posterior mean is equal to the signal. At the other end of the spectrum, when signals are uninformative (i.e. $\sigma_{\varepsilon}^{2} \rightarrow \infty$ ) the signal noise dominates overconfidence. ${ }^{10}$ Similarly, when there is no prior uncertainty about the benefit (i.e. $\sigma_{b}^{2} \rightarrow 0$ ), there is no misconception. In this case, of course, a trial would be worthless. At the other extreme, as $\sigma_{b}^{2} \rightarrow \infty$, the therapeutic misconception vanishes, again because the correct posterior mean equals the signal.

\section{B. Disappointment}

The therapeutic misconception is concerned with subjects having excessively optimistic beliefs about the benefit of participating in a trial. Disappointment exists when $E[b \mid \zeta]>b$. Selection alone is enough to induce disappointment

\footnotetext{
${ }^{10}$ An alternative formulation of overconfidence has individuals believing the signal noise to be $\hat{\sigma}_{\varepsilon}^{2}$, while the true noise is $\hat{\sigma}_{\varepsilon}^{2}+\bar{\sigma}_{\varepsilon}^{2}$. In this case, the therapeutic misconception monotonically increases with $\bar{\sigma}_{\varepsilon}^{2}$.
} 
among subjects, because individuals that face the prospect of good outcomes but receive misleadingly bad signals choose not to become subjects. If individuals are Bayesian, the fact that Bayesian updating is unbiased implies

$$
\int\left[\int_{-\infty}^{\infty} E^{*}[b \mid \zeta] d G^{*}(\zeta \mid b)-b\right] d F(b)=0
$$

so that with selection,

$$
\int\left[\frac{1}{1-G^{*}(\hat{\zeta} \mid b)} \int_{\hat{\zeta}}^{\infty} E^{*}[b \mid \zeta] d G^{*}(\zeta \mid b)-b\right] d F(b)>0 .
$$

However, an important consequence of overconfidence is that it increases the likelihood that subjects will be disappointed ex post. Figure 2 plots for each $b$ the expected difference between posterior beliefs and the true benefit for the Bayesian case $(\lambda=1)$ and for the case in which there is overconfidence $(\lambda>1)$. In both cases, the difference is declining, but at a greater rate for the Bayesian case. In each case, there exists a $b^{*}(\lambda)>1$, strictly increasing in $\lambda$, such that for any realized benefit less than $b^{*}$, subjects will on average turn out to be disappointed.

Factors affecting selection and the revision of posterior beliefs will in turn affect the average degree of disappointment. As in the previous subsection, we can calculate for the normal case that disappointment will be more widespread and profound in trials with more overconfident subjects, that impose greater personal costs on subjects, and that have intermediate values of $\sigma_{b}^{2}$ and $\sigma_{\varepsilon}^{2}$.

\section{Attenuating Therapeutic Misconception and Disappointment}

There are several ways in which therapeutic misconception and disappointment can be attenuated or, in some cases, eliminated. First, subjects could be kept completely uninformed. Doing so equates the prior and posterior 


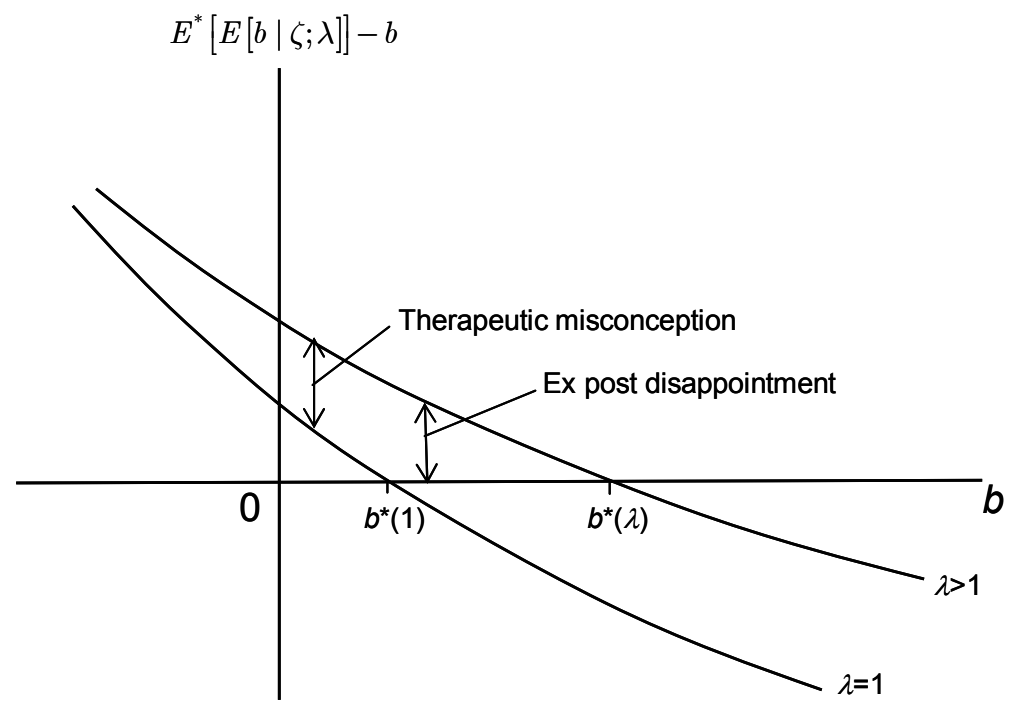

FIGURE 2. Plots of $\int_{0}^{\infty} E[b \mid \zeta] d G^{*}(\zeta \mid b)-b$

beliefs and eliminates selection, and in this case there is on average no misconception and no disappointment among subjects. Second, subjects could be kept perfectly informed. In this case, $E[b \mid \zeta] \equiv b$, so there is neither therapeutic misconception nor disappointment. Third, it may be possible to educate potential subjects so that overoptimism is reduced. Doing so will attenuate both misconception and disappointment. However, even if all overoptimism was eliminated, disappointment would continue to plague the average subject because of selection.

A fourth alternative is to pay imperfectly informed subjects. Payment will attenuate both the therapeutic misconception and disappointment because it reduces selection. Let $w$ denote the payment made to subjects, and let $b$ continue to denote the medical benefits accruing to participation. Then an individual will 
choose to become a subject if she receives a signal $\zeta>\hat{\zeta}(w)$, where $E[b \mid \hat{\zeta}(w)]=-w$. Clearly, $\hat{\zeta}(w)$ is monotonically decreasing in $w$. Hence, the higher the payment the less misconception and disappointment there will be.

If the support of the prior distribution, $F(b)$, has finite lower bound, $\underline{b}<0$, then there exists a payment, $w(\underline{b})$, increasing in $|\underline{b}|$, that eliminates selection entirely. If $\underline{b} \rightarrow-\infty$, no finite payment can eliminate selection. Nonetheless, selection - and hence the therapeutic misconception and disappointment - can be reduced. To attain any target level of misconception or disappointment, the indicated payment is greater for intermediate values of $\sigma_{b}^{2}$ and $\sigma_{\varepsilon}^{2}$, and it is increasing in $\lambda$ and $c$.

\section{Self-selection by Researchers}

Just as people do not become subjects at random, neither do researchers select the trials they conduct at random. Clinical researchers behave just as do researchers in other fields by pursuing opportunities that look most promising to them. With the great degree of specialization in research, it is perhaps inevitable that researchers in any particular area have considerably more information about promising avenues than does the profession as a whole. In this section, we show that in the presence of selection by researchers, collective equipoise in the profession as a whole is very likely to preclude collective equipoise among researchers as a group. To illustrate how selection of researchers can matter, we then show how this undermining of collective equipoise in turn undermines the rationale for designing trials to maximize statistical power.

\section{A. Selection and Equipoise}

Consider a randomized trial intended to compare an innovative treatment 
with either an existing treatment or a placebo. The outcome for patient $i$ receiving treatment $j$ is given by $q_{i j}=\mu_{j}+\varepsilon_{i}$, where the innovative treatment, $j=1$ is compared against the alternative $j=0$, and $\varepsilon_{i} \sim N\left(0, \sigma^{2}\right)$. Assume that $\mu_{0}$ is known, and let $\mu=\mu_{1}-\mu_{0}$. Collective equipoise implies that the unconditional distribution, $F(\mu)$ has mean zero. However, when the opportunity to conduct the trial is presented to a researcher it is accompanied by a signal, $\zeta \in[\underline{\zeta}, \bar{\zeta}]$, that is as usual positively correlated with $\mu$. The researcher has beliefs about $\mu$ given by the conditional distribution $F(\mu \mid \zeta)$. Her payoff from conducting the trial is zero if the new treatment turns out to be no better than the existing treatment or a placebo (i.e. if $\mu \leq 0)$, and by the increasing, concave utility function, $u(\mu)$, $u(0)=0$, otherwise.

A researcher facing a static choice about whether to conduct this trial will choose to do so regardless of the signal. In that case, there is no selection and the expected value of all trials is consistent with collective equipoise. But researchers do not select projects in a static setting. Conducting one trial precludes, or postpones, the pursuit of other perhaps more exciting opportunities. And in this dynamic setting, the choice a researcher makes about any one trial does depend on the signal she receives.

Consider the following stylized setting. A researcher can only conduct one trial, but an opportunity is presented each period with probability $\phi$. If the researcher chooses to not to pursue the current opportunity, then next period she must wait again with probability $1-\phi$, while with probability $\phi$ she receives another opportunity with signal $\zeta^{\prime}$. This is a classic optimal stopping problem. Let $\theta$ denote the discount factor. The value of being presented with an opportunity with signal $\zeta$ is 


$$
V(\zeta)=\max \left\{E[u(\mu) \mid \zeta], \theta\left[\phi E\left[V\left(\zeta^{\prime}\right)\right]+(1-\phi) \nu\right]\right\}
$$

where

$$
\nu=\theta\left[\phi E\left[V\left(\zeta^{\prime}\right)\right]+(1-\phi) \nu\right]
$$

is the value of not being presented with an opportunity. If the researcher chooses to pursue the current opportunity, she receives an expected benefit of $E[u(\mu) \mid \zeta]$. Solving (12) for $\nu$ and substituting into (11) yields

$$
V(\zeta)=\max \left\{E[u(\mu) \mid \zeta], \frac{\theta \phi E\left[V\left(\zeta^{\prime}\right)\right]}{1-\theta(1-\phi)}\right\} .
$$

There is a unique signal, $\hat{\zeta}$, such that the researcher chooses not to conduct the trial if and only if $\zeta<\hat{\zeta}$. Assuming $\left\{\zeta, \zeta^{\prime}\right\}$ are independent, $V(\zeta)=V(\hat{\zeta})=E[u(\mu) \mid \hat{\zeta}]$ for all $\zeta \leq \hat{\zeta}$. If $\zeta>\hat{\zeta}$, the researcher pursues the current opportunity and $V(\zeta)=E[u(\mu) \mid \zeta]$. Hence, $\hat{\zeta}$ satisfies

$$
E[u(\mu) \mid \hat{\zeta}] \geq \frac{\theta \phi}{1-\theta(1-\phi)}\left[\int_{-\infty}^{\hat{\zeta}} E[u(\mu) \mid \hat{\zeta}] d G(\zeta)+\int_{\zeta^{*}}^{\infty} E[u(\mu) \mid \zeta] d G(\zeta)\right] .
$$

A simple rearrangement yields the fundamental reservation equation for the expected utility of conducting a trial:

$$
E[u(\mu) \mid \hat{\zeta}] \geq \frac{\theta \phi}{1-\theta} \int_{\hat{\zeta}}^{\infty}(E[u(\mu) \mid \zeta]-E[u(\mu) \mid \hat{\zeta}]) d G(\zeta)
$$

Equation (15) allows a corner solution, when (15) is satisfied for $\hat{\zeta}=\underline{\zeta}$. This possibility is more likely when the signal is known to be noisy (so prior beliefs are not revised too drastically) or when $\theta$ or $\phi$ is small (so the option value of waiting is limited). In this case, there is no selection, and collective equipoise holds simultaneously for researchers and for the profession as a whole. In many cases, 
however, we can expect an interior solution, $\hat{\zeta}>\underline{\zeta}$, for which (15) is an equality. As the left hand side of (15) is increasing in $\hat{\zeta}$ while the right hand side is decreasing in $\hat{\zeta}$, the interior solution is unique. In this case, researchers are not in collective equipoise as selection implies that

$$
\frac{1}{1-G(\hat{\zeta})} \int_{\hat{\zeta}}^{\bar{\zeta}} E[\mu \mid \zeta] d G(\zeta)>\int_{\underline{\zeta}}^{\bar{\zeta}} E[\mu \mid \zeta] d G(\zeta)=E[\mu]=0
$$

The required expected utility to justify conducting the trial is increasing in $\theta$ and $\phi$, and hence so is $\hat{\zeta}$. Hence the deviation from collective equipoise among researchers is increasing in the rate at which new opportunities for participation arrive and in the discount factor.

To extend the analysis further, assume now that researchers can consider new opportunities after the current trial is completed. Assume that that a trial lasts $n>1$ periods, where $n$ is an i.i.d. draw. The value function in this case is given by

$$
\begin{aligned}
V(\zeta, n)=\max \left\{E[u(\mu) \mid \zeta]+\theta^{n}\left[\phi E\left[V\left(\zeta^{\prime}, n^{\prime}\right)\right]+(1-\phi) \nu\right]\right. \\
\left.\theta\left[\phi E\left[V\left(\zeta^{\prime}, n^{\prime}\right)\right]+(1-\phi) \nu\right]\right\} .
\end{aligned}
$$

Following the same steps as previously, the reservation equation satisfies

$$
E[u(\mu) \mid \hat{\zeta}(n)]=\frac{\left(\theta-\theta^{n}\right) \phi}{1-\theta} E V\left(\zeta^{\prime}, n^{\prime}\right) .
$$

As $\left\{n, n^{\prime}\right\}$ and $\left\{\zeta, \zeta^{\prime}\right\}$ are pairs of independent r.v.s and $\theta \in[0,1]$, the right hand side of (18) is strictly increasing in $n$. Hence $\hat{\zeta}(n)$ is strictly increasing in $n$, so that the deviation from collective equipoise is greater among researchers conducting long trials. 


\section{B. Selection and Statistical Power}

Clinical researchers must balance diverse concerns in the conduct of trials. On the one hand, they should be concerned that the scientific value of the trial be as great as possible. On the other, they should be concerned with the welfare of subjects. Equipoise allows researchers to avoid a potential conflict between these concerns. The scientific value of research is enhanced by, inter alia, greater statistical power of an experimental design. Equipoise allows researchers to design a trial to maximize statistical power without facing a conflict with concern for the welfare of subjects. However, when researchers are not in equipoise, the conflict is not so readily avoided. Concern for the expected welfare of subjects leads the researcher to prefer that a greater fraction of subjects receive the treatment about which the researchers is optimistic. Unfortunately, doing so reduces statistical power.

To see this, consider again the clinical trial setting described in the previous subsection, and assume again that the unconditional distribution, $F(\mu)$ has mean zero. Selection now implies that, while researcher beliefs are $F(\mu \mid \zeta)$, the domain is reduced to $\zeta \in[\hat{\zeta}, \bar{\zeta}]$, and $E[\mu]>0$ for each researcher. Finally, although the researcher has beliefs governed by Bayesian thinking, she follows norms in the profession and compares means with classical statistics.

Let $n$ denote the sample size and let $\rho$ denote the fraction of the sample assigned to the innovative treatment. The power, $\beta$, of a $t$-test for a difference in means with size five percent, as measured by the $z$ score, is

$$
z_{1-\beta}=-1.96+\frac{\sqrt{\rho(1-\rho)} \sqrt{n}|\mu|}{\sigma} .
$$

which, regardless of beliefs about $\mu$, is maximized by dividing subjects evenly 
between the two treatments. However, the researcher is concerned with balancing the expected power of the test against the expected benefit to participants, and so faces the objective function

$$
\max _{\rho}\left\{(1-\alpha) \frac{\sqrt{\rho(1-\rho)} \sqrt{n}}{\sigma} \int_{-\infty}^{\infty}|\mu| d F(\mu \mid \zeta)+\alpha \rho \int_{-\infty}^{\infty} \mu d F(\mu \mid \zeta)\right\} .
$$

The solution, $\rho^{*}$, satisfies

$$
-\frac{\left(1-2 \rho^{*}\right)}{2 \sqrt{\rho^{*}\left(1-\rho^{*}\right)}}=\frac{\alpha \sigma}{(1-\alpha) \sqrt{n}} \frac{\int \mu d F(\mu \mid \zeta)}{\int|\mu| d F(\mu \mid \zeta)} .
$$

The left (L) and right (R) hand sides of (21) are plotted in Figure 3. If there is no signal, then $E[\mu]=0$ and $\rho^{*}=0.5$. However, given a signal sufficiently promising to induce the researcher to conduct the trial in the first place, the right hand side is strictly positive, so that $\rho^{*}>0.5$. Perhaps surprisingly, increases in $\sigma$ and reductions in $n$ shift $\mathbf{R}$ up and induce the researcher to increase the fraction of subjects, $\rho^{*}$, receiving the innovative treatment. When there is little variation in outcomes across individuals or the sample size is larger, researchers get more "bang for their buck" in terms of increased power upon keeping $\rho$ close to 0.5 . Finally, a mean preserving spread in the researcher's posterior distribution raises $\int|\mu| d F(\mu \mid \zeta)$, shifts $\mathbf{R}$ downwards, and therefore reduces $\rho^{*}$. Thus, if two researchers have the same posterior mean for $\mu$, then the researcher with the greater confidence in this expectation will choose to raise the fraction of subjects receiving the innovative treatment by the greater amount.

\section{Conclusions}

Selection by participants into clinical trials may lead to ex ante therapeutic 


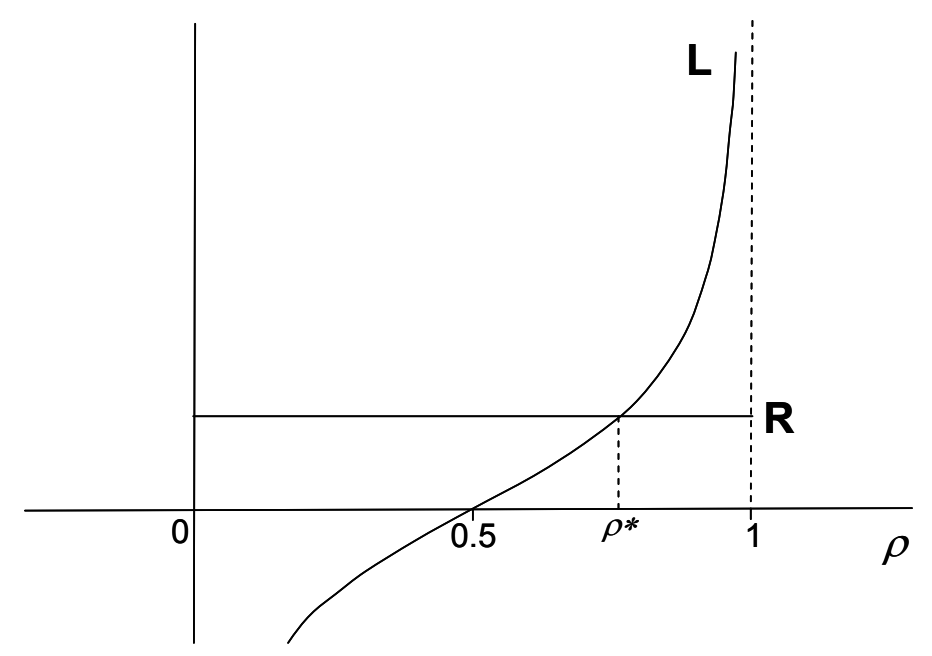

FIGURE 3. Solution to (21)

misconception and ex post disappointment on the part of subjects. Selection by researchers into clinical trials is incompatible with maintaining collective and individual equipoise, and implies that balanced randomization is unethical. In this paper, we explore both of these situations, and propose solutions for both.

For research participants, disappointment and therapeutic misconception may always exist, and may be exacerbated by individual characteristics. We find that when individuals are overconfident in the signals that they receive concerning the benefits of trial participation, therapeutic misconception and disappointment are increased. Unfortunately, little empirical evidence exists as to which other individual characteristics affect the therapeutic misconception. Lidz and Appelbaum [2002] review the literature, and report that some studies have linked age and education to understanding of trial procedures, and illness severity with attributing therapeutic goals to non-therapeutic research. However, no studies appear to directly measure a relationship between individual 
characteristics and either misconception or disappointment.

When the therapeutic misconception and/or disappointment are induced in trial participants, the trial - and research as a whole - may suffer. Individuals may drop out of trials if they become aware of their misconception, and may then hold, and spread, a distrust of clinical research. It is because of this, and the ethical requirement of respect for persons, that researchers and ethicists try to devise ways of reducing therapeutic misconception. Education and more extensive informed consent do not appear to work. Here, we propose a nontrivial payment as a means of ameliorating therapeutic misconception and disappointment. This is not an entirely new idea, as the National Institutes of Health Section on Human Subjects Research in 2002 proposed "evaluating the possibility of an inverse relationship between money for research participation and the therapeutic misconception." (Grady et al. [2002]). Findings from this research may eventually yield data that supports our claims here.

Selection by researchers when deciding which trials to conduct may also have substantial ethical and practical implications for conducting clinical trials. If, as we argue, research select into trials due to private information, and collective equipoise is an important ethical criteria for conducting a trial, then maximizing statistical power with equal samples appears unethical. This as well is not a new idea. Acknowledging that individual equipoise is unlikely in most clinical trials, others have also argued that a unbalanced sample may be useful for enhancing the ethical design of human subjects research (Avins [1998], Edwards and Braunholtz [2000]). The specific weights that one should give to current benefits for participants and future benefits to society will be difficult to calculate, and may vary with a number of characteristics of a specific research project. Such a calculation is outside of the realm of formal economic analysis, but empirical 
psychological and ethical research may reveal approximate figures.

Because of the long and continuing debates over both the therapeutic misconception and equipoise, formalization of these concepts, as we have done here, seems long overdue. The predictions generated by our model should inform future empirical research in this area.

\section{References}

Appelbaum, Paul S. (1996): "Examining the ethics of human subjects research." Kennedy Institute of Ethics Journal, 6(3):285-286.

Appelbaum, Paul S. (2002): "Clarifying the ethics of clinical research: a path toward avoding the therapeutic misconception." The American Journal of Bioethics, 2(2):22-23.

Appelbaum, Paul S., Loren Roth and Charles Lidz (1982): "The therapeutic misconception: Informed consent in psychiatric research." International Journal of Law and Psychiatry, 5(3-4):319-329.

Avins, A.L. (1998): "Can unequal be more fair? Ethics, subject allocation, and randomized clinical trials." Journal of Medical Ethics, 24(6):401-408.

Barber, Brad M., and Terrance Odean (2001): "Boys will be boys: gender, overconfidence, and common stock investment." Quarterly Journal of Economics

Berg, Jessica (1997): "Finders' fees in research protocols." American Medical News, June 22-30, p. 19.

Chard, J.A., and R.J. and Lilford (1998): "The use of equipoise in clinical trials." Social Science and Medicine, 47(7):891-898.

Compte, Olivier (2004): "Prediction errors and the winner's curse." Manuscript: CERAS, Ecole Nationale des Ponts et Chaussées.

Daniel, Kent, David Hirshleifer and Avanidar Subrahmanyam (1998): "Investor psychology and security market under- and overreactions." Journal of Finance, 53:1839-1885. 
De Long, J. Bradford, Andre Schliefer, Lawrence H. Summer and Robert J. Waldmann (1991): "The survival of noise traders in financial markets." Journal of Business, 64:1-19.

Dickert, Neal, and Christine Grady (1999): "What's the price of a research subject? Approaches to payment for research participation." New England Journal of Medicine, 341(3):198.

Dresser, Rebecca A. (2002): "The ubiquity and utility of the therapeutic misconception." Social Philosophy \& Policy 19:271-294.

Edwards, Sarah J.L. and David A. Braunholtz (2000): "Can unequal be more fair? A response to Andrew Avins." Journal of Medical Ethics, 26:179-182.

Freedman, B. (1987): "Equipoise and the ethics of clinical research." New England Journal of Medicine, 317:141-145.

Grady, Christine, et al. (2002): "Payment of research subjects." Mimeo: National Institutes for Health, Section on Human Subjects Research, Unit on Clinical Research. http://www.bioethics.nih.gov/research/humanres/payment.pdf. Accessed May 2005.

Gifford, F. (1995): "Community equipoise and the ethics of randomised clinical trials." Bioethics, 9(2):127-148.

Johnson, N., R.J. Lilford, and W. Brazier (1991): "At what level of collective equipoise does a clinical trial become ethical?" Journal of Medical Ethics, 17:30-34.

Kass, Nancy, Jeremy Sugarman, Ruth Faden, and M. Schoch-Spana (1996): "Trust: The fragile foundation of contemporary biomedical research." Hastings Center Report, 26(5):25-29.

Kerr, C., E. Robinson, A. Stevens, D. Braunholtz, S. Edwards, and R. Lilford. (2005): "Randomisation in trials: do potential trial participants understand it and find it acceptable?" Journal of Medical Ethics, 30:80-84

Kennedy, I. (1991): "The law and ethics of informed consent and randomized control trials." In I. Kennedy, ed., Treat Me Right :essays in Medical Law and Ethics, Oxford: Clarendon Publications, pp. 213-224. 
Klepper, Steven, and Peter Thompson (2005): "Spinoff entry in high-tech industries: motives and consequences." Manuscript: Carnegie Mellon University.

Kyle, Albert S., and F. Albert Wang (1997): "Speculation duopoly with agreement to disagree: can overconfidence survive the market test?" Journal of Finance, 52:20732090.

Lichtenstein, Sarah, Baruch Fischhoff and Lawrence Phillips (1982): "Calibration of probabilities" the state of the art to 1980." In Daniel Kahneman, Paul Slovic, and Amos Tversky, eds., Judgment under Uncertainty: Heuristics and Biases. Cambridge: Cambridge University Press, pp. 30-6-334.

Lidz, Charles W, and Paul Appelbaum (2002): "The therapeutic misconception: Problems and solutions." Medical Care, 40:V-55-V-63.

Lilford, Richard J. (2003): "Ethics of clinical trials from a Bayesian and decision analytic perspective: whose equipoise is it anyway?" British Medical Journal, 326:980-981.

Miller, Franklin, and Andrew Shorr (1999): "Advertising for clinical research." IRB 21(5):2.

Moreno, Jonathan, et al. (1998):"Updating protections for human subjects involved in research." Journal of the American Medical Association, 280(22):1954.

Odean, Terrance (1998): "Volume, volatility, price, and profit when all traders are above average." Journal of Finance, 53:1887-1934.

Truog, Robert, et al. (1999): "Is informed consent always necessary for randomized controlled trials?" New England Journal of Medicine, 340(10):804-807.

Yates, J. Frank (1990): Judgment and Decision Making. Englewood Cliffs, NJ: Prentice Hall. 